\title{
Herbicidas e Reguladores de Crescimento de Plantas Utilizados na Cana-de-Açúcar e sua Ação sobre Adultos de Trichogramma galloi Zucchi (Hymenoptera: Trichogrammatidae)
}

\author{
Marina de Rezende Antigo' ${ }^{1}$, Eduardo Mitio Shimbori², Daniele Fabiana Glaeser², \\ Harley Nonato de Oliveira ${ }^{\circledR}$ \& Gerado Andrade Carvalho ${ }^{3}$
}

\begin{abstract}
1. Universidade Federal da Grande Dourados, e-mail: mahrezende@hotmail.com. 2. Embrapa Agropecuária Oeste, e-mail: shimbori@gmail.com, daniglaeser@yahoo.com.br, harley.oliveira@embrapa.br (Autor para correspondência ${ }^{\bowtie}$ ). 3. Universidade Federal de Lavras, e-mail: gacarval@den.ufla.br.
\end{abstract}

\section{EntomoBrasilis 9 (1): 36-40 (2016)}

Resumo. Durante o manejo da cultura da cana-de-açúcar são utilizados diversos produtos fitossanitários que podem afetar o desempenho de parasitoides, inimigos naturais da broca-da-cana, Diatraea saccharalis (Fabricius) e consequentemente, o controle biológico da mesma. Assim, objetivou-se avaliar em laboratório o efeito de herbicidas e reguladores de crescimento de plantas utilizados na cultura da cana-de-açúcar, sobre a sobrevivência, e parasitismo de adultos do parasitoide de ovos Trichogramma galloi Zucchi, assim como sobre a emergência do parasitoide (F). Os produtos testados foram: Clomazone, Diuron + Hexazinone (herbicidas); Sulfometuron-methyl e Trinexapac-ethyl (reguladores de crescimento de plantas) nas maiores doses recomendadas à cultura. Ovos de $D$. saccharalis foram imersos durante 5 segundos em uma das caldas químicas e, após secos por 1 hora, oferecidos às fêmeas de T. galloi, totalizando 20 repetições. Avaliou-se a mortalidade e o número de ovos parasitados pela geração $\mathrm{F}_{\text {o }}$, assim como, a emergência da geração $\mathrm{F}_{1}$ de $T$. galloi, sendo os produtos classificados quanto à toxicidade, conforme percentual de redução da sobrevivência, parasitismo e emergência do parasitoide. Em relação à sobrevivência de T. galloi, número de ovos parasitados pela $\mathrm{F}_{0}$ e a emergência da $\mathrm{F}_{1}$, Clomazone e Trinexapac-ethyl foram classificados como levemente prejudicais. Diuron + Hexazinone e Sulfometuron-methyl foram inócuos em todos os parâmetros avaliados, com exceção do parasitismo, onde Diuron + Hexazinone foi classificado como levemente prejudicial. Conclui-se que, Clomazone, Diuron + Hexazinone, Trinexapac-ethyl e Sulfometuron-methyl são inócuos ou levemente prejudiciais a sobrevivência e parasitismo $\left(\mathrm{F}_{\mathrm{o}}\right)$ de adultos de T. galloi, e a emergência ( $\left.\mathrm{F}_{1}\right)$ desse parasitoide, em laboratório.

Palavras-Chave: Controle Biológico; Crambidae; Manejo Integrado de Pragas; Parasitoides; Seletividade.

\section{Herbicides and Plant Growth Regulators Used in Sugarcane and their Action on Adult Trichogramma galloi Zucchi (Hymenoptera: Trichogrammatidae)}

Abstract. During crop sugarcane management, several used pesticides can affect the performance of parasitoids, natural enemies of sugarcane borer, Diatraea saccharalis (Fabricius) and consequently the biological control of it. Thus, the objective was to evaluate in laboratory the effect of herbicides and plant growth regulators used in the sugarcane crops on survival and parasitism in adults of the eggs parasitoids, Trichogramma galloi Zucchi. The products tested were the herbicides Clomazone and Diuron + Hexazinone, and the plant regulators Sulfometuron-methyl and Trinexapacethyl, all in the higher recommended dosages for sugarcane. The eggs of D. saccharalis were immersed during 5 seconds in chemical solutions and, after dry for 1 hour, offered to T. galloi females with 20 repetitions. We evaluated the mortality and number of eggs parasitized by the $\mathrm{F}$ generation of $T$. galloi, as well as the emergence of the $\mathrm{F}_{1}$. The products were classified by their toxicity, according to the percentage of reduction in the survival, parasitism and emergence of the parasitoid. In relation to the survival of adult T. galloi, the number of eggs parasitized by $\mathrm{F}_{\mathrm{o}}$ as well as the percentage of $\mathrm{F}_{1}$ emergence, clomazone and trinexapac-ethyl were classified as slightly harmful. Diuron+hexazinone and sulfometuron-methyl were harmless in all parameters, except for parasitism, where diuron+hexazinone was classified as slightly harmful. We conclude that clomazone, diuron+hexazinone, trinexapac-ethyl and sulfometuron-methyl, are harmless or slightly harmful the survival and parasitism $\left(\mathrm{F}_{0}\right)$ of $T$. galloi adults and emergence $\left(\mathrm{F}_{1}\right)$ of this parasitoid in the laboratory.

Keywords: Biological Control; Crambidae; Integrated Pest Management; Parasitoids; Seletivity.

cultura da cana-de-açúcar ocupa área aproximada de 9,5 milhões de hectares no Brasil, com uma produção média anual de aproximadamente 678,3 milhões de toneladas (IBGE 2015). Entretanto, a produtividade pode ser afetada por uma série de fatores, com destaque para a ocorrência de insetospraga, como a broca-da-cana, Diatraea saccharalis (Fabricius) (Lepidoptera: Crambidae) (Botelho 1992; BRoglio-MicheletTi et al. 2006) que pode reduzir a produção em até $1,14 \%$, para cada 1\% de índice de infestação (PINTO et al. 2009).

Ao contrário da maioria das culturas de importância econômica onde o controle químico predomina, no caso da broca-dacana-de-açúcar, a principal medida de controle é o controle biológico por meio do parasitoide larval, Cotesia flavipes (Cameron) (Hymenoptera: Braconidae) e do parasitoide de ovos, Trichogramma galloi Zucchi (Hymenoptera: Trichogrammatidae) ambos liberados nos canaviais geralmente na fase adulta (Lima Filho \& Lima 2003).

O sucesso do controle biológico com o uso de parasitoides depende de condições bióticas e abióticas favoráveis, além de práticas de manejo adequadas, incluindo o uso de produtos fitossanitários seletivos a esses inimigos naturais (Nicholls et al. 2007). Dentre os produtos utilizados na cultura da cana

Agências de Financiamento: CAPES, CNPq e Embrapa 
destacam-se os herbicidas que são usados no manejo de plantas daninhas e os reguladores de crescimento de plantas para antecipar o processo de maturação da cana (CAPUTO et al. 2008; CARVALHo et al. 2010).

Herbicidas e reguladores de crescimento de plantas são geralmente produtos químicos sintetizados de maneira a atuar sobre a fisiologia das plantas (CAPUTO et al. 2008; CarvalHo et al. 2010). Entretanto, alguns desses produtos podem afetar diretamente a fisiologia de insetos e de outros artrópodes, podendo influenciar no manejo integrado de pragas (NoRRIs \& Kogan 2000; Dal PogetTo 2011), por prejudicar a eficiência dos inimigos naturais (MANZONI et al. 2007; NÖRNBERG et al. 2009) afetando a sobrevivência, o parasitismo e a emergência destes insetos.

Pesquisas relacionadas à seletividade de herbicidas registrados para a cultura do milho demonstraram efeitos adversos sobre Trichogramma pretiosum Riley (Hymenoptera: Trichogrammatidae), em condições de laboratório (StEFANELlo Júnior et al. 2008, 2011) e também à $T$. pretiosum e Trichogramma atopovirilia Oatman \& Platner (Hymenoptera: Trichogrammatidae) em maçã (MANzonı et al. 2006, 2007). Os herbicidas avaliados por SEBAI et al. (2012) em laboratório foram classificados como moderadamente prejudiciais ou inócuos a adultos de Trichogramma cacoeciae Marchal (Hymenoptera: Trichogrammatidae). HASSAN (1994), ao estudar o efeito de inúmeros pesticidas sobre $T$. cacoeciae, verificou que alguns herbicidas e também reguladores de crescimento de plantas foram prejudiciais para adultos desse parasitoide.

Objetivou-se avaliar o efeito de herbicidas e reguladores de crescimento de plantas sobre a sobrevivência e parasitismo de adultos de $T$. galloi, visando determinar a seletividade destes produtos sobre este parasitoide.

\section{MATERIAL E MÉTODOS}

Os testes de seletividade dos produtos fitossanitários à adultos de T. galloi foram realizados em setembro de 2011, no laboratório de Entomologia/Controle Biológico da Embrapa Agropecuária Oeste. Os produtos avaliados foram os herbicidas Clomazone $(800 \mathrm{~g} / \mathrm{L})$ e Diuron + Hexazinone $(488 \mathrm{~g} / \mathrm{Kg}+142 \mathrm{~g} / \mathrm{Kg})$, e os reguladores de crescimento de plantas, Sulfometuronmethyl (750 g/Kg) e Trinexapac-ethyl (250 g/L) (número entre parênteses refere-se à concentração do ingrediente ativo no produto comercial). Todas as soluções foram consideradas nas concentrações máximas indicadas para a cultura (maior dose do produto/menor volume calda) (AGROFIT 2015).

Os parasitoides foram obtidos do Laboratório de Controle Biológico da Embrapa. Uma fêmea adulta de T. galloi, com até $24 \mathrm{~h}$ de idade, foi individualizada em tubo de vidro $(8,5 \mathrm{~cm}$ de altura x $2,5 \mathrm{~cm}$ de diâmetro), contendo uma gotícula de mel para sua alimentação, sendo o tubo fechado com PVC laminado. Posteriormente, 60 ovos de $D$. saccharalis com até $24 \mathrm{~h}$ de idade, ovipositados em papel jornal e contados sob microscópio estereoscópico, foram imersos durante cinco segundos nas caldas químicas dos produtos fitossanitários, ou em água destilada (testemunha), e em seguida, permaneceram à temperatura ambiente de sala climatizada $\left(25 \pm 2{ }^{\circ} \mathrm{C}\right.$, UR de $\left.60 \pm 10 \%\right)$ por $1 \mathrm{~h}$. Após secos, os ovos foram oferecidos às fêmeas visando o parasitismo por um período de $24 \mathrm{~h}$, conforme a metodologia proposta por Vianna et al. (2009) e BRUgGer et al. (2010). Posteriormente, as massas de ovos de $D$. saccharalis expostas às fêmeas foram mantidas em tubos de vidro com um pedaço de algodão umidificado em água destilada para evitar a dessecação dos ovos, sendo então armazenadas em câmara climatizada tipo BOD a $23 \pm 2{ }^{\circ} \mathrm{C}$, UR de $60 \pm 10 \%$ e fotofase de $14 \mathrm{~h}$, até a emergência dos parasitoides.

Avaliou-se a mortalidade das fêmeas, assim como o parasitismo de T. galloi $\left(\mathrm{F}_{\mathrm{o}}\right)$ nos ovos tratados nas caldas químicas e a emergência dos indivíduos oriundos dos ovos tratados $\left(\mathrm{F}_{1}\right)$.

O delineamento experimental utilizado foi inteiramente ao acaso, com cinco tratamentos e 20 repetições, sendo os insetos utilizados de mesmo lote. Os dados foram submetidos à análise de variância (ANOVA) e as médias dos tratamentos comparadas à $5 \%$ de probabilidade, pelo teste de Scott-Knott usando o programa estatístico SISVAR 5.3 (FERREIRA 2011).

Os produtos testados foram enquadrados em classes toxicológicas em função da porcentagem de redução(PR)da capacidadebenéfica do parasitoide (sobrevivência, parasitismo e emergência) em relação ao tratamento testemunha (água destilada) (STERK et al. 1999), cujo cálculo foi realizado por meio da seguinte equação:

$\mathrm{PR}=100-\left(\frac{\text { Porcentagem média geral do tratamento com o produto }}{\text { Porcentagem média geral da testemunha }} \times 100\right)$

Na classe 1 foram enquadrados os produtos inócuos (redução menor que 30\%), na classe 2 os levemente prejudiciais (redução entre 30 a $79 \%$ ), na classe 3, os moderadamente prejudiciais (redução entre 80 a 99\%) e na classe 4 os prejudiciais (redução maior que $99 \%$ ). Tal classificação segue recomendações de membros da IOBC (International Organization for Biological and Integrated Control of Noxious Animals and Plants) (STERK et al. 1999).

\section{RESULTADOS E DISCUSSÃO}

Ao comparar o efeito de dois herbicidas e reguladores de crescimento de plantas sobre a mortalidade de $T$. galloi verificou-se que o herbicida Clomazone diferiu da testemunha, sendo classificado como levemente prejudicial, enquanto Diuron + Hexazinone não apresentou diferenças, sendo considerado inócuo aos adultos desse parasitoide. Já para os reguladores de crescimento de plantas, Trinexapac-ethyl diferiu, enquanto Sulfometuron-methyl não diferiu significativamente da testemunha, sendo classificados como levemente prejudicial e inócuo, respectivamente (Tabela 1).

Tabela 1. Mortalidade (\%) ( \pm EP) e porcentagem média de redução (PR) da sobrevivência de fêmeas de Trichogramma galloi (Hymenoptera: Trichogrammatidae) após o contato com ovos de Diatraea saccharalis (Lepidoptera: Crambidae) tratados com produtos fitossanitários utilizados na cana-de-açúcar, em teste de seletividade e classes de toxicidade.

\begin{tabular}{cccc}
\hline Tratamentos & \% mortalidade & PR & Classe $^{1}$ \\
\hline Testemunha & $0,0 \pm 0,00 \mathrm{a}$ & - & - \\
Clomazone & $45,0 \pm 11,41 \mathrm{~b}$ & 45,0 & 2 \\
Diuron + Hexazinone & $10,0 \pm 6,88 \mathrm{a}$ & 10,0 & 1 \\
Trinexapac-ethyl & $35,0 \pm 10,94 \mathrm{~b}$ & 35,0 & 2 \\
Sulfometuron-methyl & $10,0 \pm 6,88 \mathrm{a}$ & 10,0 & 1 \\
\hline
\end{tabular}

Médias seguidas pela mesma letra minúscula na coluna não diferem entre si pelo teste de Scott-Knott a $5 \%$ de probabilidade.

'Índice de toxicidade segundo a IOBC: Classe 1 - inócuos (redução menor que $30 \%$ ), classe 2 - levemente prejudiciais (redução entre 30 a $79 \%$ ), classe 3 - moderadamente prejudiciais (redução entre 80 a 99\%); classe 4 - prejudiciais (redução maior que 99\%) (STERK et al. 1999).

Conforme constatado, Clomazone e Trinexapac-ethyl foram levemente prejudiciais à T. galloi. Por serem produtos que afetam a fisiologia de plantas, esperava-se que os mesmos não afetassem a sobrevivência desses inimigos naturais. No entanto, Norris \& Kogan (2000); Dal Pogetto (2011) relataram que agrotóxicos de modo geral, incluindo alguns herbicidas podem apresentar efeitos tóxicos (diretos e indiretos) sobre artrópodes, incluindo insetos benéficos. Os efeitos tóxicos diretos podem ocorrer em função da toxicidade das moléculas aos organismos ou sobre a influência que estes exercem sobre os parâmetros de 
desenvolvimento dos insetos (DAL PogetTo 2011). Ressalta-se que não foram encontrados na literatura trabalhos relacionados aos efeitos específicos dos produtos avaliados sobre a sobrevivência de parasitoides, mas como foram testadas às doses máximas recomendadas para a cultura da cana, as altas concentrações podem ter contribuído para essa toxicidade dos produtos ao parasitoide.

Em relação ao parasitismo de $T$. galloi, com exceção de Sulfometuron-methyl, que não apresentou diferenças significativas e foi classificado como inócuo, todos os demais produtos avaliados diferiram da testemunha e foram classificados como levemente prejudicais (Tabela 2).

Tabela 2. Número médio ( \pm EP) de ovos de Diatraea saccharalis (Lepidoptera: Crambidae) parasitados por fêmea de Trichogramma galloi (Hymenoptera: Trichogrammatidae) da geração maternal $\left(\mathrm{F}_{0}\right)$ e porcentagem média de redução (PR) do parasitismo em teste de seletividade.

\begin{tabular}{cccc}
\hline Tratamentos & $\begin{array}{c}\text { Ovos } \\
\text { parasitados } \\
\left(\mathbf{n}^{\mathbf{0}}\right)\end{array}$ & PR & Classe $^{\mathbf{1}}$ \\
\hline Testemunha & $22,6 \pm 2,17 \mathrm{a}$ & - & - \\
\hline Clomazone & $11,2 \pm 2,4 \mathrm{ob}$ & 50,3 & 2 \\
\hline Diuron + Hexazinone & $15,0 \pm 2,5 \mathrm{ob}$ & 33,5 & 2 \\
\hline Trinexapac-ethyl & $10,0 \pm 2,11 \mathrm{~b}$ & 55,5 & 2 \\
\hline Sulfometuron-methyl & $34,9 \pm 2,17 \mathrm{a}$ & 0 & 1 \\
\hline
\end{tabular}

Médias seguidas pela mesma letra minúscula na coluna não diferem entre si pelo teste de Scott-Knott a 5\% de probabilidade.

'Índice de toxicidade segundo a IOBC: Classe 1 - inócuos (redução menor que $30 \%$ ), classe 2 - levemente prejudiciais (redução entre 30 a 79\%), classe 3 - moderadamente prejudiciais (redução entre 80 a 99\%); classe 4 - prejudiciais (redução maior que 99\%) (STERK et al. 1999).

As diferenças nas taxas de parasitismo, comparado a testemunha, para os produtos classificados como levemente prejudiciais, pode ser devido à hipótese de que estes produtos tenham uma ação de repelência. ANTIGo et al. (2013), ao estudarem a repelência de produtos fitossanitários sobre adultos de T. galloi, também verificaram menores taxas de parasitismo em ovos tratados com os produtos Clomazone, Diuron + Hexazinone e Trinexapacethyl quando comparados a ovos não tratados, assim como não relataram diferenças no parasitismo para ovos tratados com Sulfometurom-methyl. BASTOS et al. (2006) observaram que Clomazone afetou negativamente o parasitismo de $T$. pretiosum em ovos de Sitotroga cerealella (Oliver) (Lepidoptera: Gelechiidae) e Anagasta kuehniella (Zeller) (Lepidoptera: Pyralidae), mas não o classificou quanto à toxicidade. O percentual de parasitismo constatado por esses autores foi de $61,2 \%$ para ovos de $S$. cerealella e de 15,2\% para ovos de A. kuehniella.

Clomazone e Trinexapac-ethyl diferiram da testemunha, apresentando maiores percentuais de redução da emergência de T. galloi $\left(\mathrm{F}_{1}\right)$, sendo classificados como levemente prejudiciais. Já Diuron + Hexazinone e Sulfometuron-methyl foram considerados inócuos (Tabela 3).

A redução nas taxas de emergência de T. galloi para clomazone e trinexapac-ethyl pode estar relacionada a hipótese de que durante o parasitismo, a exposição das fêmeas a esses produtos e a inserção do ovipositor pelo parasitoide no ovo contaminado de $D$. saccharalis afetaram o desenvolvimento dos imaturos no hospedeiro. Ressalta-se que Oliveira et al. (2014) observaram que Clomazone não afetou a emergência de T. galloi em ovos de $D$. saccharalis quando este produto foi aplicado sobre o ovo contendo o parasitoide na fase de pupa. CARMO et al. (2010) também verificaram que Clomazone não afetou a emergência de T. pretiosum em ovos de A. kuehniella (inócuo), quando esses continham o parasitoide em fase de pupa. Segundo esses autores, estando o parasitoide na fase de pupa e a mesma ainda protegida dentro do córion do hospedeiro, essa fase seria considerada a mais resistente à ação dos produtos fitossanitários. Por outro lado, neste trabalho, adultos de T. galloi foram expostos ao produto, o que pode explicar o maior índice de toxicidade (levemente prejudicial) ao parasitoide, que não se encontra protegido e, consequentemente, efeitos negativos podem ocorrer no parasitismo da $F_{0}$ e também na emergência da $F_{1}$

Tabela 3. Porcentagem de emergência ( \pm EP) de Trichogramma galloi $(\mathrm{F})$ e porcentagem média de redução (PR) da emergência em ovos de Diatraea saccharalis tratados com produtos fitossanitários.

\begin{tabular}{cccc}
\hline Tratamentos & \% emergência & PR & Classe $^{\mathbf{1}}$ \\
\hline Testemunha & $63,4 \pm 4,26 \mathrm{a}$ & - & - \\
Clomazone & $13,5 \pm 5,88 \mathrm{c}$ & 78,6 & 2 \\
Diuron + Hexazinone & $55,3 \pm 5,61 \mathrm{a}$ & 12,7 & 1 \\
Trinexapac-ethyl & $32,4 \pm 6,95 \mathrm{~b}$ & 48,9 & 2 \\
Sulfometuron-methyl & $59,2 \pm 4,51 \mathrm{a}$ & 6,6 & 1 \\
\hline
\end{tabular}

Médias seguidas pela mesma letra minúscula na coluna não diferem entre si pelo teste de Scott-Knott a 5\% de probabilidade.

'Índice de toxicidade segundo a IOBC: Classe 1 - inócuos (redução menor que 30\%), classe 2 - levemente prejudiciais (redução entre 30 a 79\%), classe 3 - moderadamente prejudiciais (redução entre 80 a 99\%); classe 4 - prejudiciais (redução maior que 99\%) (STERK et al. 1999).

Assim como Clomazone, Trinexapac-ethyl foi levemente prejudicial à emergência de T. galloi $\left(\mathrm{F}_{1}\right)$, reduções na emergência para esse produto também foram observadas por ANTigo et al. (2013), ao aplicarem este produto sobre ovos de D. saccharalis. Já a inocuidade de Diurom+hexazinona e Sulfumeturom-methyl, corrobora com os resultados obtidos por ANTIGO et al. (2013), que também não observaram reduções na emergência desse parasitoide.

Como os produtos avaliados foram considerados inócuos ou levemente prejudicais em laboratório não é necessária a continuidade dos testes em outras condições visto que, segundo a IOBC, quando produtos fitossanitários testados em suas dosagens máximas são classificados como inócuos ou levemente prejudiciais a adultos em laboratório, não é necessário testes subsequentes em condições de semicampo (avaliação da persistência do produto) e de campo (MANzoni et al. 2006), pois a tendência é uma redução da toxicidade para estas condições devido a degradação dos compostos (NÖRNBERG et al. 2011).

De maneira geral, o herbicida Diuron + Hexazinone foi mais seletivo à T. galloi, quando comparado à Clomazone. Para os reguladores de crescimento, Sulfometuron-methyl apresentou melhores resultados de seletividade (inócuo), comparado à Trinexapac-ethyl (levemente prejudicial).

Conclui-se que Clomazone, Diuron + Hexazinone, Trinexapacethyl e Sulfometuron-methyl são inócuos ou levemente prejudiciais à sobrevivência e parasitismo $\left(\mathrm{F}_{\mathrm{o}}\right)$ de adultos de T. galloi, assim como à emergência $\left(\mathrm{F}_{1}\right)$ desse parasitoide, em condições de laboratório.

\section{AGRADECIMENTOS}

A CAPES, CNPq e Embrapa pelo apoio financeiro.

\section{REFERÊNCIAS}

AGROFIT 2015. Ministério da Agricultura Pecuária e Abastecimento, Sistema de Agrotóxicos Fitossanitários. Disponível em: <http://agrofit.agricultura.gov.br/agrofit cons/principal agrofit cons >. [Acesso em: 20.07.2015].

Antigo, M.R., H.N. Oliveira, G.A. Carvalho \& F.F. Pereira, 2013. Repelência de produtos fitossanitários usados na cana-deaçúcar e seus efeitos na emergência de Trichogramma galloi. Revista Ciência Agronômica, 44: 910-916. 
Bastos, C.S., R.P. Almeida \& F.A. Suinaga, 2006. Selectivity of pesticides used on cotton (Gossypium hirsutum) to Trichogramma pretiosum reared on two laboratoryreared hosts. Pest Management Science, 62: 91-98. DOI: http://dx.doi.org/10.1002/ps.1140.

Botelho, P.S.M., 1992. Quinze anos de controle biológico da Diatraea saccharalis utilizando parasitoides. Pesquisa Agropecuária Brasileira, 27: 255-262, Edição Especial.

Broglio-Micheletti, S.M.F., A.J.N. Santos \& J.L. Pereira-barros, 2006. Ação de alguns produtos fitossanitários para adultos de Trichogramma galloi Zucchi, 1988 (Hymenoptera: Trichogrammatidae). Ciência e Agrotecnologia, 30: 1051-1055. DOI: http://dx.doi.org/10.1590/S1413-70542006000600001.

Brugger, K.E., P.G. Cole, I.C. Newman, N. Parker, B. Scholz, P. Suvagia, G. Walker \& T.G. Hammond, 2010. Selectivity of chlorantraniliprole to parasitoid wasps. Pest Management Science, 6: 1075-1081. DOI: http://dx.doi.org/10.1002/ ps.1977.

Caputo, M.M., E.G.F. Beauclair, M.A. Silva \& S.M.S. Piedade, 2008. Resposta de genótipos de cana-de-açúcar à aplicação de indutores de maturação. Bragantia, 67: 15-23. DOI: http://dx.doi.org/10.1590/Sooo6-87052008000100002.

Carmo, E.L., A.F. Bueno, R.C.O.F, S.S. Vieira, M.M.P. Goulart, M.M. \& T.R. Carneiro, 2010. Seletividade de produtos fitossanitários utilizados na cultura da soja para pupas de Trichogramma pretiosum Riley, 1879 (Hymenoptera: Trichogrammatidae). Arquivos do Instituto Biológico, 77: 283-290.

Carvalho, F.T., R.M. Castro, R.I. Otsubo \& F.A.R. Pereira, 2010. Controle de dez espécies daninhas em cana-deaçúcar com o herbicida mesotrione em mistura com ametryn e metribuzin. Planta Daninha, 28: 585-590. DOI: http://dx.doi.org/10.1590/S0100-83582010000300015.

Dal Pogetto, M.H.F.A., 2011. Impacto de herbicidas sobre a biologia e controle de Spodoptera frugiperda (Lepidoptera: Noctuidae) na cultura do milho. Tese (Doutorado em Agonomia ) - Universidade Estadual Paulista. 75 p.

Ferreira, D.F., 2011. Sisvar: a computer statistical analysis system. Ciência e Agrotecnologia, 35: 1039-1042.

Hassan, S.A., 1994. Comparison of three different laboratory methods and one semi-field test method to assess the side effects of pesticides on Trichogramma cacoeciae Marchal (Hymenoptera: Trichogrammatidae). Bulletin OILB/SROP, 17: 133-141.

IBGE. Levantamento Sistemático da Produção Agrícola: junho de 2015.81p. RiodeJaneiro, 2015. Disponívelem: < ftp://ftp.ibge. gov.br/Producao Agricola/Levantamento Sistematico da Producao Agricola [mensal]/Fasciculo/2015/lspa 201506. pdf $>$. [Acesso em: 24.07.2015].

Lima Filho, M. \& J.O.G. Lima, 2003. Diatraea saccharalis (Fabr.) em cana-de-açúcar na região norte do Estado do Rio de Janeiro: flutuação populacional e parasitismo de ovos por Trichogramma spp. Revista Universidade Rural, 22: 33-44.

Manzoni, C.G., A.D. Grützmacher, F.P. Giolo, W.R. Härter \& C. Müller, 2006. Seletividade de agrotóxicos usados na produção integrada de maçã para adultos de Trichogramma pretiosum. Pesquisa Agropecuária Brasileira, 41: 1461-1467. DOI: http://dx.doi.org/10.1590/So100-204X2006001000002.

Manzoni, C.G., A.D. Grützmacher, F.P. Giolo, W.R. Härter, R.V. Castilhos \& M.D.F. Paschoal, 2007. Seletividade de agroquímicos utilizados na produção integrada de maçã aos parasitóides Trichogramma pretiosum Riley e Trichogramma atopovirilia Oatman \& Platner (Hym.: Trichogrammatidae). Bioassay, 2: 1-11. DOI: http://dx.doi.org/10.14295/BA.v2.0.50.

Nicholls, C.I., M.A. Altieri \& L. Ponti, 2007. Controle biológico de pragas através do manejo de agroecossistemas. Brasília, DF: Ministério do Desenvolvimento Agrário, Departamento de Assistência Técnica e Extensão Rural, 2007. 31 p.
Nörnberg, S.D., A.D. Grützmaker, A. Kovaleski, E.S. Camargo \& R.A. Pasini, 2009. Toxicidade de agrotóxicos utilizados na produção integrada de maçã a Trichogramma pretiosum Riley, 1879 (Hymenoptera: Trichogrammatidae) em condições de laboratório. Revista Brasileira de Agrociência, 15: 49-56.

Nörnberg, S.D., A.D. Grützmaker, A. Kovaleski, J.A. Finatto \& M.D.F. Paschoal, 2011. Persistência de agrotóxicos utilizados na produção integrada de maçã a Trichogramma pretiosum. Ciência e Agrotecnologia, 35: 305-313. DOI: http://dx.doi.org/10.1590/S1413-70542011000200011.

Norris, R.F. \& M. Kogan, 2000. Interactions between weeds, arthropods pests, and their natural enemies in managed ecosystems. Weed Science, 48:94-158.

Oliveira, H.N., M.R. Antigo, G.A. Carvalho, D.F. Glaeser, 2014. Effect of selectivity of herbicides and plant growth regulators used in sugarcane crops on immature stages of Trichogramma galloi (Hymenoptera: Trichogrammatidae). Planta Daninha, 32:125-131. DOI: http://dx.doi.org/10.1590/So100-83582014000100014.

Pinto, A.S., P.S.M. Botelho \& H.N. Oliveira, 2009, Guia ilustrado de pragas e insetos benéficos da cana-de-açúcar. Piracicaba: $\mathrm{CP} 2,160 \mathrm{p}$.

Sebai, O.A., F. Mohamed, A.E. Osama, 2012. Side-effect of certain herbicides on egg parasitoid Trichogramma evanescens (West.) (Hymenoptera: Trichogrammatidae). Academic Journal of Entomology, 5: 1-10.

Stefanello Júnior, G.J., A.D. Grützmacher, D.D. Grützmacher, C.A.B Lima, D.O Dalmozo \& M.D.F. Paschoal, 2008. Seletividade de herbicidas registrados para a cultura do milho a adultos de Trichogramma pretiosum (Hymenoptera: Trichogrammatidae). Planta Daninha, 26: 343-351. DOI: http://dx.doi.org/10.1590/So100-83582008000200010.

Stefanello Júnior, G.J., A.D. Grützmacher, R.A. Pasini, C. Bonez, D.C. Moreira \& D. Spagnol, 2011. Seletividade de herbicidas registrados para a cultura do milho aos estádios imaturos de Trichogramma pretiosum (Hymenoptera: Trichogrammatidae). Planta Daninha, 29: 1069-1077. Edição especial. DOI: http://dx.doi.org/10.1590/S0100-83582011000500014.

Sterk, G., S.A. Hassan, M. Baillod, F. Bakker, F. Bigler, S. Blümel, H. Bogenschütz, E. Boller, B. Bromand, J. Brun, J.N.M. Calis, J. Coremans-Pelseneer, C. Duso, A. Garrido, A. Grove, U. Heimbach, H. Hokkanen, J. Jacas, J. Lewis, L. Moreth, L. Polgar, L. Roversti, L. Samsoe-Petersen, B. Sauphanor, L. Schaub, A. Stäubli, J.J. Tuset, A.Vainio, M. Van de Veire, G. Viggiani, E. Viñuela \& H. Vogt, 1999. Results of the seventh joint pesticide testing programme carried out by the IOBC/ WPRS-Working Group 'Pesticides and Beneficial Organisms'. BioControl, 44: 99-117.

Vianna, U.R., D. Pratissoli, J.C. Zanuncio, E.R. Lima, J. Brunner, F.F. Pereira, J.E. Serrão, 2009. Insecticide toxicity to Trichogramma pretiosum (Hymenoptera: Trichogrammatidae) females and effect on descendant generation. Ecotoxicology, 18: 180-186. DOI: http://dx.doi.org/10.1007/s10646-008-0270-5.

Recebido em: 21.ix.2015

Aceito em: 14.ii.2016 
Como citar este artigo:

Antigo, M.R., E.M. Shimbori, D.F. Glaeser, H.N. Oliveira, G.A. Carvalho, 2016. Herbicidas e Reguladores de Crescimento de Plantas Utilizados na Cana-de-Açúcar e sua Ação sobre Adultos de Trichogramma galloi Zucchi (Hymenoptera: Trichogrammatidae). EntomoBrasilis, 9 (1): $36-40$. Acessível em: doi:10.12741/ebrasilis.vgi1.556
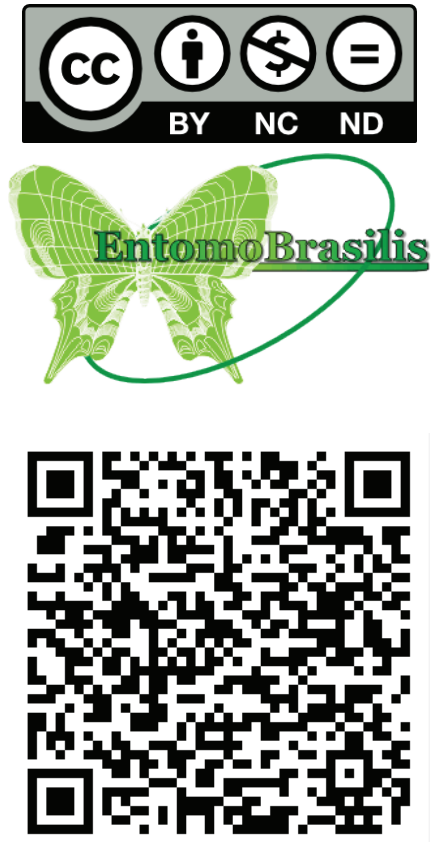